\title{
TRANSFERENCIA DE CALOR TRANSIENTE EM UMA BARRA METÁLICA: EXPERIMENTO MULTIDISCIPLINAR EM ENGENHARIA QUÍMICA
}

\author{
G. P. F. GASPAR, A. S. VIANNA JR, J. A. W. GUT \\ Universidade de São Paulo, Escola Politécnica, Dep.de Engenharia Química \\ E-mail para contato: jorgewgut@usp.br
}

\begin{abstract}
RESUMO - Este trabalho apresenta uma proposta de experimento didático multidisciplinar para engenharia química envolvendo as disciplinas de Transferência de Calor e de Análise de Processos. O fenômeno observado é de dissipação de calor por uma barra metálica (aleta) em estado transiente. As atividades envolvidas são de modelagem do processo com solução analítica para o estado estacionário e numérica para o transiente, ajuste do modelo estacionário por estimação de parâmetros, simulação dinâmica por diferenças finitas e comparação e discussão de resultados. Um microcontrolador de hardware e software livres de baixo custo teve bom desempenho na aquisição e transmissão de dados. Experimentos foram realizados em cinco temperaturas diferentes para fonte quente e os resultados de predição do modelo ajustado foram satisfatórios e coerentes. A união dos conhecimentos experimental, teórico e numérico tem por objetivo fornecer uma visão sistêmica da análise dos Fenômenos de Transporte, indicando os passos necessários para se realizar a correta modelagem de um fenômeno natural ao estudante.
\end{abstract}

\section{INTRODUÇÃO}

$\mathrm{Na}$ Indústria de Processos Químicos é comum encontrar superfícies quentes que trocam calor com o ar. A utilização de superfícies estendidas (aletas) aumenta a área de contato para a troca convectiva com a função de elevar a taxa de transferência de calor ou reduzir a temperatura superficial. Para estudar este fenômeno, neste experimento considera-se uma longa barra de seção transversal circular dissipando calor para o ar ambiente a partir de uma fonte quente.

O experimento proposto neste trabalho visa elucidar a importância desse fenômeno no projeto de equipamentos de troca térmica, além de quantificar as perdas de calor nesses equipamentos, as quais são inerentes ao seu design. Um dos objetivos almejados com este trabalho é ilustrar a interdisciplinaridade entre o conhecimento dos Fenômenos de Transporte e dos métodos numéricos.

Esse laboratório didático forneceu um ponto de conexão entre as disciplinas PQI2302 - Fenômenos de Transporte II e PQI2403 - Análise de Processo. Uma contribuição para a integralização do currículo didático da Engenharia Química na Escola Politécnica da USP. 


\section{MODELAGEM MATEMÁTICA}

Considere uma barra metálica de seção transversal circular (comprimento $L$ e raio $R$ ) usada como difusor de calor (aleta) de uma fonte quente. A energia térmica entra na base da aleta, cuja temperatura é $T_{b}$, difunde pela barra e transfere-se para o ar por convecção com coeficiente $h$ e temperatura $T_{\infty}$. Aplica-se ao volume de controle da barra a equação diferencial de conservação de energia térmica:

$$
\frac{\partial T}{\partial t}+\vec{v} \cdot \vec{\nabla} T-\alpha \nabla^{2} T-\frac{\dot{q}_{V}}{\rho \cdot C p}=0
$$

em que $T$ é a temperatura, $t$ é o tempo, $\vec{v}$ é a velocidade, $\alpha=k / \rho . C p$ é a difusividade térmica, $k$ é a condutividade térmica, $\rho$ é a densidade, $C p$ é o calor específico à pressão constante e $\dot{q}_{V}$ é a taxa volumétrica de geração de calor. Considerando transporte unidirecional ao longo da barra, tem-se o seguinte modelo adimensional:

$$
\frac{\partial \theta}{\partial F_{O}}-\frac{\partial^{2} \theta}{\partial x^{2}}+(m . L) \theta=0
$$

em que $\theta^{*}=\left(T-T_{\infty}\right) /\left(T_{b}-T_{\infty}\right)$ é a temperatura adimensional, $F o=\alpha . t / L^{2}$ é o número de Fourier (tempo adimensional), $x^{*}=x / L$ é a posição axial adimensional. $\mathrm{O}$ único parâmetro deste modelo é o grupo adimensional $m . L$, sendo que $m^{2}=2 . h / k . R$.

O modelo pode ser resolvido analiticamente para as condições do estado estacionário. A condição de contorno para a posição $x^{*}=0$ é $\theta^{*}=1$, enquanto que a condição de contorno na extremidade da barra depende da hipótese adotada. Segundo Incropera et al. (2008), se m.L $>2,65$, a temperatura na ponta da aleta $\left(x^{*}=1\right)$ pode ser considerada como sendo $T_{\infty}$. Neste caso, pode-se assumir a condição de $\theta^{*}=0$ resultado no seguinte perfil de temperatura:

$$
\theta^{*}=\frac{\operatorname{senh}\left[m \cdot L \cdot\left(1-x^{*}\right)\right]}{\operatorname{senh}(m \cdot L)}
$$

Para a resolução do modelo transiente na Eq. (1), foram usadas as mesmas condições de contorno do modelo estacionário, a condição inicial de $\theta^{*}=0$ e foi adotado o método de diferenças finitas para frente para discretização do primeiro tempo da equação e o método de diferenças centrada de segunda ordem para o segundo termo da equação. Como resultado, tem-se uma forma explícita para calcular a temperatura no próximo instante de tempo em função do perfil de temperatura no instante "atual":

$$
\theta_{j}^{*_{n+1}}=\theta_{j}^{*_{n}}+\left(\theta_{j+1}^{*_{n}}-2 \cdot \theta_{j}^{*_{n}}+\theta_{j-1}^{*_{n}}\right)\left(\frac{\Delta F O}{\Delta x^{*_{2}}}\right)-(m \cdot L)^{2} \cdot \theta_{j}^{*_{n}} \cdot \Delta F O
$$


Na equação (4), o sobrescrito $n$ indica o instante de tempo discreto e o subscrito $j$ indica a posição discreta. Para a solução numérica é importante verificar o critério de estabilidade de $\left(\Delta F_{O} / \Delta x^{* 2}\right) \leq 0.5$ (Constantinides e Mostoufi, 1999).

\section{MATERIAL E MÉTODOS}

O experimento foi montado utilizando-se um banho térmico RTE-211, o qual possui um controlador PID interno para manter a temperatura constante e cuja função é ser a fonte quente na base da barra (aleta). Utilizou-se uma barra de aço com diâmetro de $1,10 \mathrm{~cm}$ e 100 $\mathrm{cm}$ de comprimento, dos quais $12 \mathrm{~cm}$ estavam imersos no banho e os outros $88 \mathrm{~cm}$ expostos ao ar ambiente. Foi utilizada uma folha de isopor para isolar a superfície do banho do ar ambiente e evitar que o vapor gerado interferisse com o experimento. A barra encontrava-se inicialmente à temperatura ambiente.

A captação dos dados foi feita através de um microcontrolador Arduino, o qual possui um slot para SDCard, no qual os dados dos sensores foram salvos. Foram utilizados cinco sensores de temperatura do tipo termistores digitais, modelo LM35 com precisão de $0,2{ }^{\circ} \mathrm{C}$, dispostos nas posições $x^{*}=0,023,0,136,0,244,0,489$ e 0,972 .

O Arduino cria uma string com as leituras do tempo decorrido e das temperaturas dos cinco sensores; essa string era salva no SDCard. Paralelamente, esses dados foram enviados para um computador via porta USB para leitura em tempo real. Essas leituras foram acessadas através de um aparelho celular também, utilizando-se um aplicativo de compartilhamento de tela, o qual permite que o monitoramento seja feito online. O monitoramento foi feito dentro e fora das dependências da USP.

Foram realizados cinco ensaios com temperaturas diferentes de banho. Foram utilizadas as temperaturas de $60,65,70,75$ e $80{ }^{\circ} \mathrm{C}$. Os ensaios tiveram 60 minutos de duração, tempo que foi estipulado como suficiente para se atingir o regime estacionário.

\section{TRATAMENTO DE DADOS}

A atividade didática começa com uma visita dos alunos ao laboratório para verificação do equipamento e elucidação do procedimento experimental. Na sequência, em sala de aula é feita a atividade de desenvolvimento do modelo matemático, sua adimensionalização e discretização do mesmo. Esta atividade tem como objetivo deduzir as Equações (2), (3) e (4) e faz parte do relatório da experiência.

Como atividade após a aula, os grupos fizeram o ajuste do modelo matemático, a simulação do modelo dinâmico e a comparação de resultados, conforme descrito na sequência.

\subsection{Ajuste do Modelo Estacionário}

O único parâmetro do modelo do processo é m.L, conforme Equações (2) e (3). Optouse por determinar este parâmetro a partir do estado estacionário. Para o intervalo de tempo em que se constatou o estado estacionário foram calculadas temperaturas médias e desvios. A 


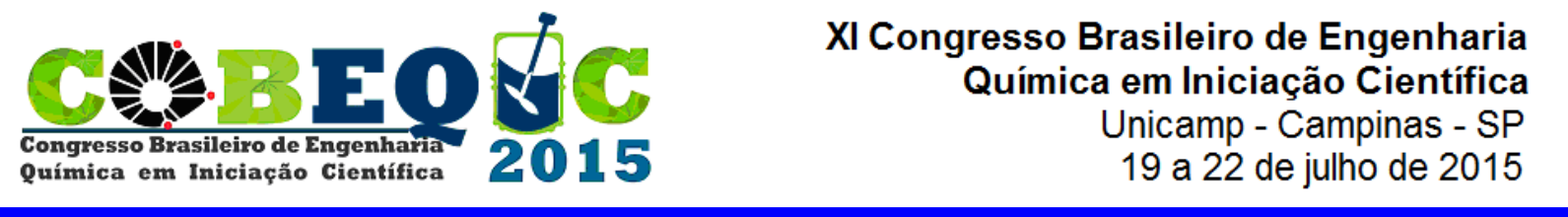

temperatura na base da aleta $\left(T_{b}\right)$ foi tratada também como um parâmetro do modelo já que verificou-se uma diferença em relação à temperatura do banho térmico. A minimização do erro quadrático de predição do modelo na Equação (2) para a temperatura nas cinco posições foi conduzida pelo método GRG - Generalized Reduced Gradient não linear por meio da ferramenta Solver do software Excel (Microsoft). Instruções para uso desta ferramenta foram passadas aos alunos. A partir do valor ajustado de m.L foi calculado o coeficiente de convecção ao ar ao redor da barra vertical.

\subsection{Simulação Dinâmica}

Definindo passos adequados para $x^{*}$ e $F o$, a simulação dinâmica do fenômeno foi feita em uma planilha Excel (Microsoft), na qual o modelo adimensional discretizado na Equação (4) foi aplicada respeitando-se as condições de contorno adimensionalizadas impostas para a resolução do modelo. Resultados foram comparados com os dados experimentais das posições dos cinco sensores de temperatura.

\section{RESULTADOS E DISCUSSÃO}

\subsection{Ajuste do Modelo Estacionário}

A Tabela 1 sumariza os resultados do ajuste do modelo estacionário com os valores estimados de $m . L$ e de $T_{b}$. A Figura 1 apresenta como exemplo de ajuste o experimento a 70 ${ }^{\circ} \mathrm{C}$, em que pode-se notar uma ótima correlação. Propriedades termofísicas do aço na temperatura médias dos experimentos foram obtidas de Incropera et al. (2008).

O valor do parâmetro m.L não teve variação significativa entre experimentos, sendo obtido um valor médio $m . L=7,3 \pm 0,3$. O coeficiente convectivo determinado para o ar foi $h$ $=8,9 \pm 0,6 \mathrm{~W} / \mathrm{K} \cdot \mathrm{m}^{2}$. Uma queda significativa de temperatura na base da aleta em relação à temperatura do banho foi observada $\left(\Delta T_{b}\right.$ na Tabela 1$)$. $O$ experimento pode ser melhorado com a monitoração desta temperatura.

Tabela 1 - Parâmetros ajustados nas condições estacionárias.

\begin{tabular}{ccccc}
\hline Banho & $m . L$ & $T_{b}\left({ }^{\circ} \mathrm{C}\right)$ & $\Delta T_{b}\left({ }^{\circ} \mathrm{C}\right)$ & $h\left(\mathrm{~W} / \mathrm{K} \cdot \mathrm{m}^{2}\right)$ \\
\hline $60^{\circ} \mathrm{C}$ & 7,63 & 50,9 & 9,1 & 9,6 \\
$65^{\circ} \mathrm{C}$ & 6,93 & 53,3 & 11,7 & 7,9 \\
$70^{\circ} \mathrm{C}$ & 7,37 & 57,8 & 12,2 & 9,0 \\
$75^{\circ} \mathrm{C}$ & 7,37 & 60,1 & 14,9 & 9,0 \\
$80^{\circ} \mathrm{C}$ & 7,43 & 64,4 & 15,6 & 9,1 \\
\hline
\end{tabular}


Figura 1 - Exemplo de ajuste do modelo estacionário (linha) aos dados experimentais (pontos) do banho a $70^{\circ} \mathrm{C}$.

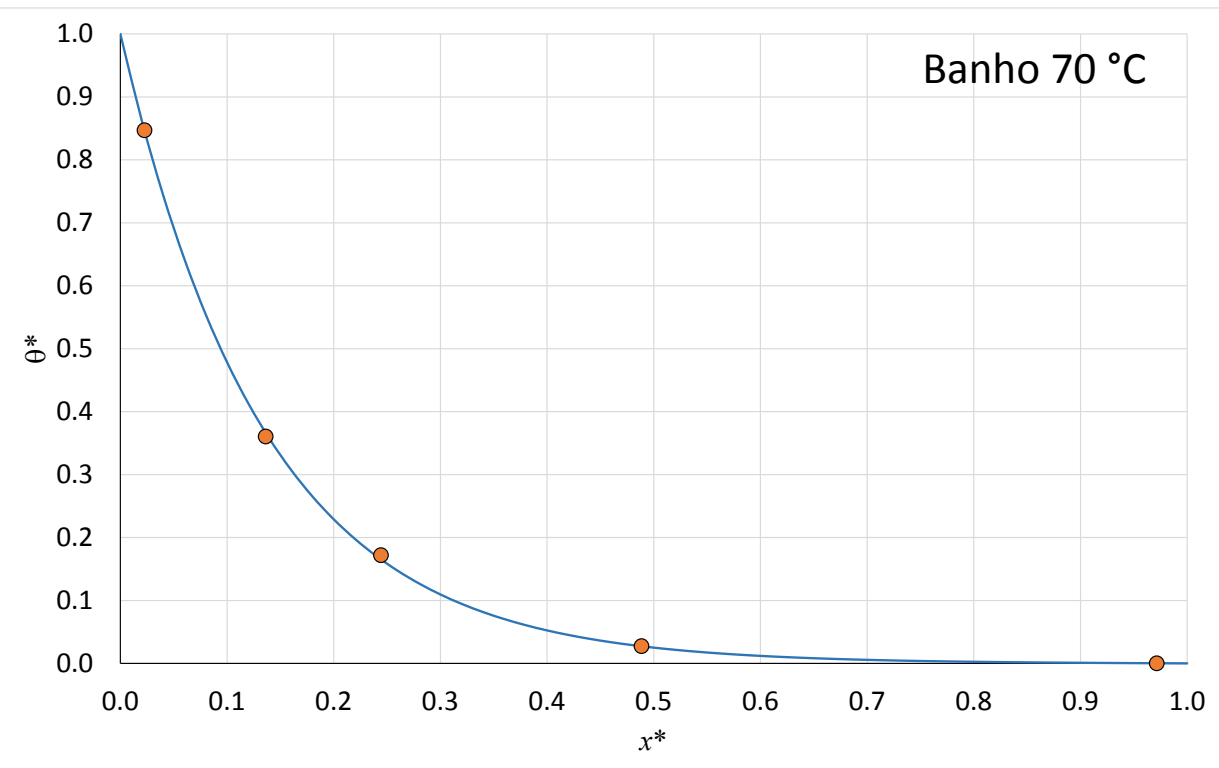

\subsection{Simulação Dinâmica}

A planilha de cálculo foi organizada distribuindo o perfil de temperatura ao longo da barra nas colunas, começando pela condição inicial na primeira linha. Nas linhas abaixo incrementa-se o valor de Fourier com o cálculo a partir da Equação (4). Nas colunas dos extremos são fornecidas as condições de contorno.

Figura 2 - Comparação entre resultados de simulação dinâmica do modelo ajustado e dados experimentais para a condição de banho a $70{ }^{\circ} \mathrm{C}$.

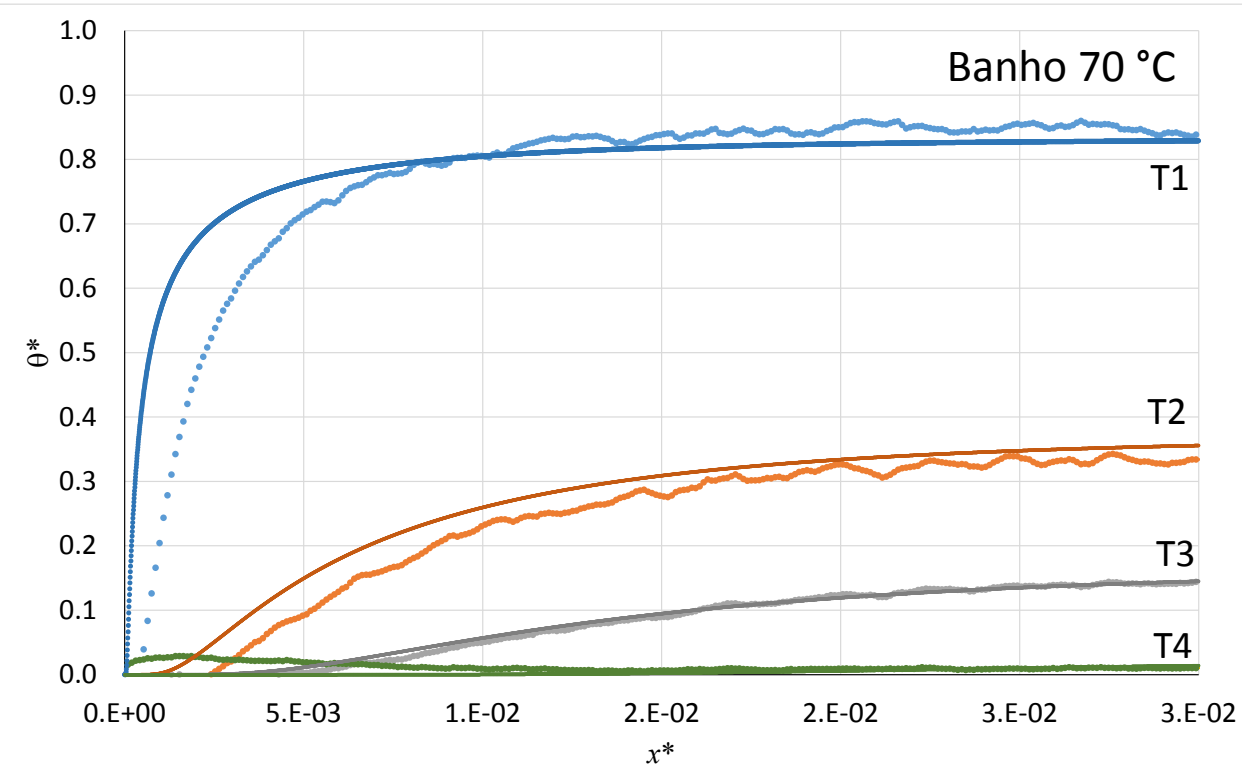




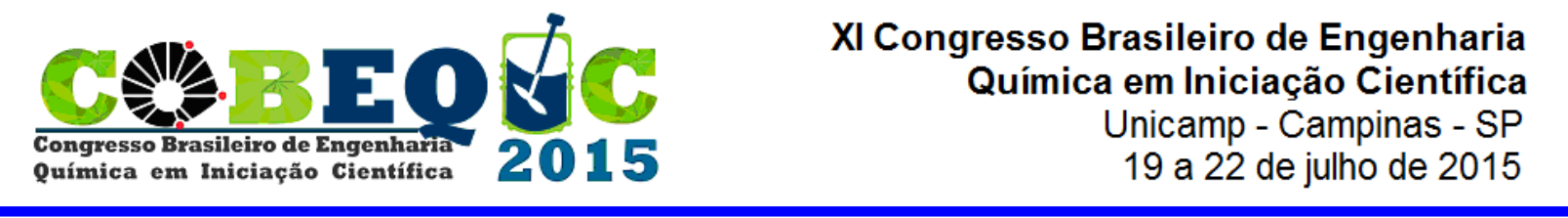

Como exemplo a Figura 2 apresenta os resultados de simulação dinâmica do modelo ajustado comparados com os dados experimentais para a condição de $70^{\circ} \mathrm{C}$. Os resultados são satisfatórios e nota-se apenas um problema de inércia em que o modelo tem uma resposta dinâmica mais rápida do que os dados experimentais, provavelmente devido ao tempo de resposta dos sensores de temperatura. O mesmo comportamento foi observado para as outras temperaturas. Outra fonte de erro pode ser o perfil radial de temperatura na barra, que foi desprezado na modelagem, sendo que os sensores medem a temperatura superficial.

\section{CONCLUSÕES}

O experimento proposto foi aplicado aos alunos do $3^{\circ}$ ano da Engenharia Química da EPUSP em 2015 e possibilitou o desenvolvimento de habilidades de modelagem de processos, ajuste de modelos e simulação dinâmica usando o software Excel.

O Arduino captou os dados de forma precisa e satisfatória, comprovando seu papel como uma ferramenta barata, flexível e de fácil acesso para o registro de dados em experimentos didáticos. É possível realizar-se a expansão para a transferência de momento e de massa, mediante a aquisição de sensores propícios para tais tarefas.

O ajuste do modelo no estado estacionário foi consistente e a estimativa dos parâmetros foi realizada com sucesso. A concordância entre os dados experimentais e o modelo teórico foi adequada. A simulação numérica no estado transiente apresentou convergência numérica e representou os dados experimentais adequadamente, comprovando a eficácia da estratégia numérica adotada para a modelagem do fenômeno estudado.

\section{REFERÊNCIAS}

ASSAF, J.M.; GIORDANO, R.C.; DUARTE FILHO, O.B.; GIORDANO, R.L.C. Tópicos de Laboratório Didático em Fenômenos de Transporte. São Carlos, SP, 1983.

CONSTANTINIDES, A.; MOSTOUFI, N. Numerical Methods for Chemical Engineers with MATLAB Applications. Upper Saddle River, NJ, 1999.

INCROPERA, F.O.; DEWITT, D.P.; BERGMAN, T.L.; LAVINE, A.S. Fundamentos de Transferência de Calor e de Massa. LTC, Rio de Janeiro, 2008. 